\title{
Identification, characterization and heparin binding capacity of a spore-wall, virulence protein from the shrimp microsporidian, Enterocytozoon hepatopenaei (EHP)
}

Pattana Jaroenlak ${ }^{1,2}$, Dominic Wiredu Boakye ${ }^{3}$, Rapeepun Vanichviriyakit ${ }^{2,4}$, Bryony A. P. Williams ${ }^{3}$, Kallaya Sritunyalucksana ${ }^{5,6}$ and Ornchuma Itsathitphaisarn ${ }^{1,2^{*}}$

\begin{abstract}
Background: The microsporidian Enterocytozoon hepatopenaei (EHP) is a spore-forming, intracellular parasite that causes an economically debilitating disease (hepatopancreatic microsporidiosis or HPM) in cultured shrimp. HPM is characterized by growth retardation and wide size variation that can result in economic loss for shrimp farmers. Currently, the infection mechanism of EHP in shrimp is poorly understood, especially at the level of host-parasite interaction. In other microsporidia, spore wall proteins have been reported to be involved in host cell recognition. For the host, heparin, a glycosaminoglycan (GAG) molecule found on cell surfaces, has been shown to be recognized by many parasites such as Plasmodium spp. and Leishmania spp.

Results: We identified and characterized the first spore wall protein of EHP (EhSWP1). EhSWP1 contains three heparin binding motifs (HBMs) at its N-terminus and a Bin-amphiphysin-Rvs-2 (BAR2) domain at its C-terminus. A phylogenetic analysis revealed that EhSWP1 is similar to an uncharacterized spore wall protein from Enterospora canceri. In a cohabitation bioassay using EHP-infected shrimp with naïve shrimp, the expression of EhSWP1 was detected by RT-PCR in the naïve test shrimp at 20 days after the start of cohabitation. Immunofluorescence analysis confirmed that EhSWP1 was localized in the walls of purified, mature spores. Subcellular localization by an immunoelectron assay revealed that EhSWP1 was distributed in both the endospore and exospore layers. An in vitro binding assay, a competition assay and mutagenesis studies revealed that EhSWP1 is a bona fide heparin binding protein.
\end{abstract}

Conclusions: Based on our results, we hypothesize that EhSWP1 is an important host-parasite interaction protein involved in tethering spores to host-cell-surface heparin during the process of infection.

Keywords: EHP, Enterocytozoon hepatopenaei, Spore wall protein, SWP, Heparin, Heparin binding protein

\section{Background}

Microsporidia are obligate, intracellular, spore-forming parasites and currently considered as a sister group to fungi [1]. Microsporidia are important pathogens that infect a wide range of animal hosts from beneficial invertebrate to vertebrate species $[2,3]$. Since the discovery of

\footnotetext{
* Correspondence: ornchuma.its@mahidol.ac.th

'Department of Biochemistry, Faculty of Science, Mahidol University, Bangkok, Thailand

${ }^{2}$ Center of Excellence for Shrimp Molecular Biology and Biotechnology (Centex Shrimp), Faculty of Science, Mahidol University, Bangkok, Thailand

Full list of author information is available at the end of the article
}

the first microsporidian Nosema bombycis in silkworms in the nineteenth century [4], it remains the cause of a fatal disease referred to as Pébrine that causes economic losses in the sericulture industry [5, 6]. Enterocytozoon hepatopenaei (EHP) is a close evolutionarily relative of Enterocytozoon bieneusi and other human-infecting microsporidia in the genus Encephalitozoon that cause life-threatening diarrhea in immunocompromized humans [7]. In aquatic animals, infection of microsporidia in fish leads to reduction in growth rate and productivity [8], and this is true also for EHP in shrimp [9]. 
Microsporidia display many unique cellular and genetic characteristics. At the cellular level, microsporidia lack peroxisomes and a typical Golgi structure [10, 11]. Their mitochondria are structurally and functionally reduced into organelles called mitosomes [12, 13]. Their genomes are remarkably compact due to the loss of genes in metabolic pathways and reduction in intergenic spaces [14]. The 2.3 Mbp genome of E. intestinalis is the smallest eukaryotic genome known to date [15]. In addition, microsporidia have developed a characteristic invasion mechanism that involves the polar tube and the spore wall [16]. At the first step of infection, the spore wall proteins are capable of interacting with host cell glycosaminoglycans (GAGs) [17, 18]. Under suitable conditions, the polar tube is extruded to pierce the host cell membrane. This process rapidly occurs in less than 2 milliseconds $[11,19]$. The polar tube then serves as a conduit to transfer an infectious sporoplasm into the host cell to begin the parasitic, intracellular phase of the life-cycle [11].

The spore walls of microsporidia consist of two layers, a proteinaceous electron dense exospore layer and a chitinous electron lucent endospore layer [20]. Many spore wall proteins (SWPs) are found in these layers [21]. They participate in the host cell recognition process and provide structural support for the spore wall [17, 21, 22]. SWPs have been extensively characterized for the genera Nosema and Encephalitozoon. These include NbSWP5, NbSWP11, NbSWP12, NbSWP16, NbSWP25 and NbS WP26 from N. bombycis [22-27], EcEnP1, EcEnP2 and chitin deacetylase (EcCDA) from E. cuniculi [28, 29], and EiEnP1 from E. intestinalis [18]. Recently, Antonospora locustae SWP2 (AlocSWP2) has been shown to be involved in sporulation [30].

Hepatopancreatic microsporidiosis (HPM) in cultivated shrimp is characterized by slow growth and wide size variation, making the causative agent $E$. hepatopenaei (EHP) an economically important pathogen for shrimp farmers [31, 32]. EHP was initially reported as a new, undescribed microsporidian in hepatopancreatic tissue of the black tiger shrimp Penaeus monodon in Thailand in 2004 [33], but it was not characterized and named as a new species until 2009 [34]. Thus, it was an endemic pathogen that was also able to cause disease in the exotic Pacific-white shrimp P. vannamei [35] that replaced $P$. monodon as the dominant and most economically important shrimp species cultivated in Thailand. Currently, EHP is known to occur widely in Asia (e.g. Thailand, China, India, Vietnam, Indonesia and Malaysia) and it has been reported more recently from Venezuela [34, 36-38]. In Thailand, EHP is now the third most serious problem for shrimp farmers after white spot disease (WSD) caused by white spot syndrome virus and acute hepatopancreatic necrosis disease (AHPND) caused by unique Vibrio isolates that produce Pir-like toxins [9].

Since EHP is a threat to the global shrimp industry, a better understanding of its infection mechanisms and virulence is urgently needed to facilitate the development of preventative and therapeutic strategies. Previously, a cohabitation assay revealed that EHP can be horizontally transmitted via water in shrimp cultivation ponds [39]. Thus, any treatment or management protocol that would stop or interfere with transmission would constitute an effective control measure. However, knowledge of how EHP interacts with the host is still poorly understood. This study therefore aimed at a better understanding of the process. From whole genome sequencing of EHP [40], the spore wall protein EhSWP1 was first identified and its gene sequence was used to develop a more specific PCR detection method called SWPPCR [31]. Here, we functionally characterize EhSWP1, show that it contains three heparin binding motifs (HBMs) and one Bin-amphiphysin-Rvs-2 (BAR2) domain, that it is localized in the exospore and endospore layers, and that interacts with heparin via its HBMs. We hypothesize that EHP uses this recognition process to initiate host cell infection, and we hope that this understanding may lead to identification of vulnerable targets for development of preventative and therapeutic methods to control EHP in the shrimp aquaculture industry.

\section{Methods}

\section{Shrimp and EHP specimens}

With permission from the farm owners to collect specimens for this study from their properties, EHP-infected P. vannamei $(7-10 \mathrm{~g})$ were collected from commercial shrimp farms in Thailand. Hepatopancreata of EHPinfected shrimp were dissected as previously described [31] to obtain spores for purification by discontinuous Percoll gradient centrifugation [40]. The purified spores were washed with sterile distilled water and stored at room temperature.

\section{Bioinformatics analysis}

In this study, we used predicted proteins encoded by the genomes of 23 microsporidian species (Enterospora canceri, Enterocytozoon hepatopenaei, Hepatospora eriocheir, Hepatospora eriocheir canceri, Anncaliia algerae, Ordospora colligata, Trachipleistophora hominis, Spraguea lophii, Vittaforma corneae, Encephalitozoon romaleae, Vavraia culicis, Edhazardia aedis, Encephalitozoon hellem Swiss, Encephalitozoon hellem ATCC, Nematocida parisii ERTm1, Nematocida parisii ERTm3, Nematocida sp. ERTm2, Nematocida sp. ERTm6, Enterocytozoon bieneusi, Encephalitozoon intestinalis, Encephalitozoon cuniculi, Nosema bombycis and Nosema ceranae). These were downloaded from public databases NCBI and 
MicrosporidiaDB. Ortholog clusters in which these proteins belonged were identified by initially querying the proteins from all 23 microsporidian genomes against their own database by using BLASTP with an e-value cut-off of 1e-03 [41]. An ortholog prediction program, ORTHOMCL on its default settings, was then used to convert the BLASTP output into ortholog clusters [42]. Phylogenetic assessment of the ortholog groups in which EHP SWPs were grouped was performed as follows. The proteins in the two ortholog groups in which EHP SWPs were clustered were first aligned with the online MAFFT program using the L-INS-I iterative refinement setting and then trimmed with GBLOCKS with less stringent settings (allowing smaller final blocks, gap positions in the final blocks and less strict flanking positions). A Bayesian inference method was also used to infer the phylogenetic relationship between the proteins in the ortholog clusters. Here, the trimmed alignment was passed to the online MR BAYES tool on the CIPRES online portal. MR BAYES was run using an LG+GAMMA model and default settings [43]. Subsequent phylogenetic analyses performed on the SWP12 clade were performed following the same protocols as explained above. Although EHP00_1468 did not cluster with any microsporidian protein in our ORTHOMCL analyses, we included it in our phylogenetic analyses as it had $98 \%$ identity to EHP00_350 in initial BLASTP analyses.

Conserved domains of proteins were predicted with MOTIF SCAN (http://www.genome.jp/tools/motif/). MOTIF SCAN searches protein sequences against a PFAM library of Hidden Markov Models (HMMs). To further assess the conservation of BAR2 domains within proteins in the SWP ortholog clusters, a pairwise alignment with the EMBOSS STRETCHER tool (https://www.ebi.ac.uk/ Tools/psa/emboss_stretcher/) of each protein against the PFAM BAR2 consensus sequence was performed. This is the consensus alignment sequence of seed proteins used by PFAM for the construction of the BAR2 HMM. The complete PFAM seed library for various functional domains can be downloaded from ftp://ftp.ebi.ac.uk/pub/databases/Pfam/current_release/Pfam-A.seed.gz. Phosphorylation site prediction was carried out by SCANPROSITE tool (http://prosite.expasy.org/prosite.html). NETNGLYC (http://www.cbs.dtu.dk/services/ NetNGlyc/) and NETOGLYC (http://www.cbs.dtu.dk/ services/NetOGlyc/) were used to predict $\mathrm{N}$ - and Oglycosylation sites, respectively.

\section{Reverse transcription PCR (RT-PCR) analysis}

To achieve EHP infections, naïve, uninfected, test $P$. vannamei were co-habitated with EHP-infected $P$. vannamei as previously described [39]. Briefly, naïve P. vannamei shrimp were kept in tanks containing $150 \mathrm{l}$ artificial seawater (Mariscience Co. Ltd, Bangkok, Thailand) at 25 ppt and $28{ }^{\circ} \mathrm{C}$ with a basket cage containing EHP-infected $P$. vannamei in the center of the tank. At 0, 5, 7, 9, 11 and 20 days after cohabitation, shrimp were collected and their hepatopancreatic tissue was aseptically removed for RNA extraction. Total RNA was extracted using Ribozol RNA extraction reagent (Amresco, Philadelphia, USA) and used as template RNA in reverse transcription reactions employing ImPromp-II reverse transcriptase (Promega, Wisconsin, USA) to produce cDNA using an oligo-dT primer. cDNA was subsequently used as the template for standard PCR with Green PCR master mix containing Taq DNA polymerase (Biotechrabbit, Hennigsdorf, Germany). The full-length EhSWP1 gene was amplified by specific primer pairs, EHP_SWP01_F; 5'--ATA TCC ATG GGC ATG TTA GAA GAT GCA AAG-3' and EHP_SWP01_R; 5'-ATA TCT CGA GAG AAA ATT TTT CAA GGT G-3'. Specific primer pairs for the actin gene of $P$. vannamei (PvActin) were used as an internal control (Actin_F; 5'-CCT CGC TGG AGA AGT CCT AC3' and Actin_R; 5'-TGG TCC AGA CTC GTC GTA CTC-3') [31, 44]. The PCR protocol for both EhSWP1 and PvActin was as follows: denaturation at 95 ${ }^{\circ} \mathrm{C}$ for 5 min followed by 30 cycles of $30 \mathrm{~s}$ denaturation at $95{ }^{\circ} \mathrm{C}, 30 \mathrm{~s}$ annealing at $55^{\circ} \mathrm{C}$ and $45 \mathrm{~s}$ extension at $68{ }^{\circ} \mathrm{C}$, with a final extension for $5 \mathrm{~min}$ at $68^{\circ} \mathrm{C}$. The expected PCR amplicons were $687 \mathrm{bp}$ and $401 \mathrm{bp}$ for EhSWP1 and PvActin, respectively. The amplicons were analyzed by $1.5 \%$ agarose gel electrophoresis with ethidium bromide staining.

\section{Molecular cloning, expression, and purification of recombinant EhSWP1}

The complete ORF of EhSWP1 (687 bp) was amplified from cDNA obtained from the hepatopancreas of EHPinfected shrimp (GenBank accession no. MG015710). PCR conditions were the same as previously described in the RT-PCR analysis section. The gene was inserted between NcoI and XhoI restriction sites of the pET28 expression vector (Novagen, Queensland, Australia) to generate a pET28a_SWP1 that was transformed into Escherichia coli BL21 Star (DE3). Positive clones were analyzed by restriction endonuclease analysis and confirmed by DNA sequencing (Macrogen, South Korea). A selected positive clone was grown in Luria-Bertani (LB) medium and induced with $0.4 \mathrm{mM}$ IPTG (isopropyl $\beta$ D-1-thiogalactopyranoside) at $37{ }^{\circ} \mathrm{C}$ for $4 \mathrm{~h}$. Bacterial cells were harvested by centrifugation at $14,000 \times g$ at $4^{\circ}$ $\mathrm{C}$ for $10 \mathrm{~min}$.

To purify recombinant EhSWP1, a bacterial cell pellet was re-suspended with $1 \times$ PBS and broken by sonication. After that, the mixture was centrifuged at $14,000 \times$ $g$ at $4{ }^{\circ} \mathrm{C}$ for $15 \mathrm{~min}$. The supernatant was collected and mixed with protein lysis buffer $\left(50 \mathrm{mM} \mathrm{NaH} \mathrm{NO}_{4}, 300\right.$ $\mathrm{mM} \mathrm{NaCl}, 10 \mathrm{mM}$ imidazole; $\mathrm{pH}$ 8) prior to loading 
onto a $\mathrm{Ni}^{2+}$-NTA affinity column (Qiagen, Hilden, Germany). Protein and $\mathrm{Ni}^{2+}$-beads were incubated for 1 $\mathrm{h}$ at $4{ }^{\circ} \mathrm{C}$. Then, the column was washed with 10 column volumes of wash buffer $\left(50 \mathrm{mM} \mathrm{NaH} \mathrm{PO}_{4}, 300 \mathrm{mM}\right.$ $\mathrm{NaCl}, 20 \mathrm{mM}$ imidazole; $\mathrm{pH}$ 8). The purified recombinant EhSWP1 was eluted with elution buffer $(50 \mathrm{mM}$ $\mathrm{NaH}_{2} \mathrm{PO}_{4}, 300 \mathrm{mM} \mathrm{NaCl}, 250 \mathrm{mM}$ imidazole; $\mathrm{pH}$ 8). All protein fractions were analyzed by $12.5 \%$ SDS-PAGE. Protein concentrations were measured using Bradford reagent (BioRad, California, USA). The purified recombinant EhSWP1 was dialyzed against $1 \times$ PBS at $4{ }^{\circ} \mathrm{C}$ overnight.

Polyclonal antibody production and Western blot analysis To produce a polyclonal antibody against EhSWP1, purified recombinant EhSWP1 was sent to a commercial antibody production facility (Singapore Advanced Biologics, Singapore) to immunize rabbits. After the third immunization, rabbit sera containing anti-EhSWP1 antibody were collected and specificity of anti-EhSWP1 antibody was tested by Western blot analysis.

For Western blot analysis, purified recombinant EhSWP1 was separated by $12.5 \%$ SDS-PAGE and transferred to a nitrocellulose membrane. The membrane was blocked with blocking solution (5\% skim milk in $1 \times$ PBS) for $1 \mathrm{~h}$ at room temperature (RT) followed by incubation with 1:2000 antiEhSWP1 antibody or naïve rabbit serum as a negative control in blocking solution for $1 \mathrm{~h}$ at RT. After six washes with PBST buffer $(1 \times$ PBS, $0.05 \%$ Tween 20$)$, 1:3000 goat anti-rabbit IgG conjugated with alkaline phosphatase enzyme (GAR-AP) was applied for $1 \mathrm{~h}$ at RT and later washed with PBST buffer three times. Finally, colorimetric signals were developed by BCIP/NBT phosphatase substrate (Millipore, Massachusetts, USA).

\section{Immunofluorescence analysis (IFA)}

Purified EHP spores were added onto poly-lysine coated slides and dried at RT overnight. The spores were fixed with 4\% paraformaldehyde at RT for 15 min followed by washing with $1 \times$ PBS three times and permeabilized with $1 \%$ Triton X-100 at RT for $30 \mathrm{~min}$. Next, the spores were blocked with blocking reagent (10\% normal goat serum, $5 \%$ bovine serum albumin in $1 \times$ PBS) at RT for $90 \mathrm{~min}$ prior to incubation with 1:100 anti-EhSWP1 antibody in blocking reagent at RT for $3 \mathrm{~h}$. The negative control group was incubated with naïve rabbit serum. After six washes, 1:200 goat anti-rabbit antibody conjugated with Alexa 488 (GAR-Alexa488) was added and incubated at RT for 1 h. 1:2000 TO-PRO-3 dye was used to stain nuclei for $5 \mathrm{~min}$ at RT. Finally, slides were mounted with $50 \%$ glycerol. The fluorescence signals were examined using a confocal laser scanning microscope (Olympus FV10i-DOC).

\section{Immunoelectron analysis (IEM)}

Purified EHP spores and EHP-infected hepatopancreatic tissue were fixed with $4 \%$ paraformaldehyde and $0.5 \%$ glutaraldehyde in $0.1 \mathrm{M}$ sodium cacodylate buffer $\mathrm{pH}$ 7.2 for $1 \mathrm{~h}$ at RT and then rinsed with $1 \times$ PBS four times. The samples were dehydrated with a graded ethanol series including 50\%, 75\% and 100\% for 15 min each step followed by permeabilizing and embedding in LRwhite (Electron Microscopy Sciences, Pennsylvania, USA). LR-white was polymerized at $65{ }^{\circ} \mathrm{C}$ overnight. Next, ultrathin sections were placed onto 300-mesh nickel grids. For immunostaining, the grids were blocked with blocking solution ( $1 \%$ bovine serum albumin, $0.02 \%$ $\mathrm{NaN}_{3}, 5 \%$ normal goat serum in $1 \times$ PBS) for $2 \mathrm{~h}$ at RT and incubated with 1:10 anti-EhSWP1 antibody in blocking solution for $2 \mathrm{~h}$ at RT. For the negative control group, naïve rabbit serum was used instead of antiEhSWP1 antibody. After six washes with 1× PBS, 1:100 anti-rabbit IgG conjugated with $10 \mathrm{~nm}$ gold particles (Sigma-Aldrich, Massachusetts, USA) in blocking solution was applied onto the grids for $1 \mathrm{~h}$ at RT and then washed with distilled water. Finally, the grids were counterstained using 4\% uranyl acetate for 2 min and gold particles were examined under a Hitachi H7100 transmission electron microscope (TEM) at an accelerating voltage of $100 \mathrm{kV}$.

\section{Site-directed mutagenesis of EhSWP1}

Basic amino acid residues of all three HBMs found in EhSWP1 gene were mutated into glycine or serine using a gene synthesis facility (Synbio Technologies, USA). EhSWP1 $(\mathrm{B} \rightarrow \mathrm{G})$ contained the following mutations: R11G, K12G, K14G, K15G, R35G, K36G, R38G, K62G, H63G, H65G and H66G, while EhSWP1 $(\mathrm{B} \rightarrow \mathrm{S})$ contained mutations R11S, K12S, K14S, K15S, R35S, K36S, R38S, K62S, H63S, H65S and H66S. After that, mutated EhSWP1 genes were subcloned into the pET28a expression vector (Novagen, Queensland, Australia). Protein expression and purification were followed as previously described for EhSWP1 WT.

\section{Heparin bead binding and competition assays}

Purified recombinant EhSWP1 $(20 \mu \mathrm{g})$ or $20 \mu \mathrm{g}$ of bovine serum albumin (Sigma-Aldrich, Massachusetts, USA) were mixed with $50 \mu \mathrm{l}$ of pre-equilibrated heparinsepharose beads (50\% slurry) with $1 \times$ PBS (GE Healthcare, Buckinghamshire, UK) at $4{ }^{\circ} \mathrm{C}$ for $1 \mathrm{~h}$ with radial rotation. For the heparin competition assay, various concentrations $(0.1,1,10$ and $100 \mathrm{mg} / \mathrm{ml})$ of porcine heparin sodium salt (Sigma-Aldrich, Massachusetts, USA) were mixed with recombinant EhSWP1 prior to incubation with heparinsepharose beads. The beads were then washed three times with $1 \times$ PBS (5 min incubation in each washing step). Proteins were eluted with elution buffer $(2 \mathrm{M} \mathrm{NaCl}$ in $1 \times$ 
PBS). All protein fractions were visualized by $12.5 \%$ SDSPAGE with Coomassie blue staining. To quantify the level of heparin binding, the intensity of the protein band was quantified using Scion Image software (Version 4.0). Level of heparin binding in the group without competitor $(0$ $\mathrm{mg} / \mathrm{ml}$ heparin group) was used for normalization.

\section{Statistical analysis}

The percentages of heparin binding were expressed as means \pm standard error of the mean (SEM). The difference between each heparin concentration was tested using one-way ANOVA.

\section{Results}

\section{Identification and characterization of EhSWP1}

To better understand the pathogenesis of EHP, a search for its potential virulence factors was carried out by analyzing the EHP genome [40] and categorizing genes according to their functions (Table 1). Putative EHP virulence factors included genes involved in host cell invasion, spore attachment, energy parasitism and host cell manipulation. To infect their host cells, microsporidia have been reported to utilize SWPs as a recognition system $[17,45]$. Herein, we describe identification of a spore wall protein, EhSWP1 (EHP00_686). The fulllength coding sequence of EhSWP1 is 687 bp encoding a deduced protein of 228 amino acids (GenBank accession no. MG015710), with a molecular mass of $27 \mathrm{kDa}$ and a theoretical isoelectric point of 8.45 .

\section{Phylogenetic analysis of EhSWP1}

An initial NCBI word search for SWP in the genomic assembly of EHP identified proteins with the following accession numbers OQS53864.1 (EHP00_686), OQS55031.1 (EHP00_944), OQS55055.1 (EHP00_1468) and OQS5342 2.1 (EHP00_350). In this study, we focused on EHP00_686, which we named EhSWP1. Our orthology analyses revealed that EhSWP1 (EHP00_686) and EHP00_350 were in a different ortholog cluster from EHP00_944 (Fig. 1). Interestingly, EHP00_1468 did not cluster with any other

Table 1 Putative virulence factors of EHP

\begin{tabular}{ll}
\hline Function & Gene \\
\hline Host cell invasion and spore & Polar tube proteins (PTPS) \\
Sttachment & Spore wall proteins (SWPS) \\
& Endochitinases \\
& Chitin synthases \\
Energy parasitism & ADP/ATP transporters \\
Host cell manipulation & Mitogen-activated protein kinases \\
& Transferases \\
& Splicing machineries \\
\hline
\end{tabular}

microsporidian protein used in this analysis despite having a 98\% identity to EHP00_350 in our BLASTP search results. Bayesian inference (BI) analyses resulted in a tree that had representative proteins from the two ortholog clusters in two distinct clades (Fig. 1). The clade in which EhSWP1, EHP00_350 and EHP00_1468 clustered contained other microsporidian proteins that were predominantly annotated as SWP12, whereas EHP00_944 was grouped within a clade containing proteins that were predominantly annotated as SWP7. Both SWP12 and SWP7 were previously described in Nosema bombycis $[24,46]$ and they were used as the name of the clades in this study. The phylogenetic relationship between these clades was however poorly supported statistically in both Bayesian and maximum likelihood (ML) analyses (Fig. 1). Apart from Nematocida species, all other microsporidian species used in this analysis were represented by at least a single protein in both the SWP12 and SWP7 clades (Fig. 2).

An initial search for functional domains in proteins belonging to the SWP12 clade showed that some of them encoded a Bin-amphiphysin-Rvs-2 (BAR2) domain. Unlike proteins in the SWP12 clade, a scan for functional domains for proteins in the SWP7 clade showed that they did not share a common functional domain. When aligned against the consensus sequence of BAR2 HMM seed sequences, proteins in the SWP12 clade showed amino acid similarity ranging between $20-29 \%$. The BAR2 domain of Saccharomyces cerevisiae protein YP148 that was one of the seed sequences used in the construction of the BAR2 HMM was $29 \%$ similar to the consensus sequence (data not shown). Proteins belonging to $V$. corneae and members of the family Encephalitozoonidae displayed the highest amino acid similarity (Fig. 2). Contrary to MOTIF SCAN results that predicted the BAR2 domains of most SWP12 clade proteins to be located in their C-terminus, amino acid pair-wise alignment analyses showed that the BAR2 domain spanned the entire length of these proteins.

A regular expression search predicted all proteins in the SWP12 clade to encode, at least, a single heparin binding motif (HBM) whereas only M896_121080 (Ordospora colligata), EDEG_03348 (Edhazardia aedis), NBO_63g0026 (Nosema bombycis) and ECANGB1_2681 (Enterospora canceri) in the SWP7 clade encoded heparin binding motifs. In this study, three HBMs were identified at the N-terminus of EhSWP1 (EHP00_686). The position of the first XBBXBBX HBM was conserved only in the family Enterocytozoonidae whereas that of the second XBBXBX HBM was conserved among most but not all microsporidian species (Fig. 2). Interestingly, the position of the third XBBXBX HBM was conserved only in EhSWP1 and ECANGB1_2216. EHP00_350 and EHP00_1468 were the only proteins in this analyses that contained the XBBBXXBX HBM signature sequence. 


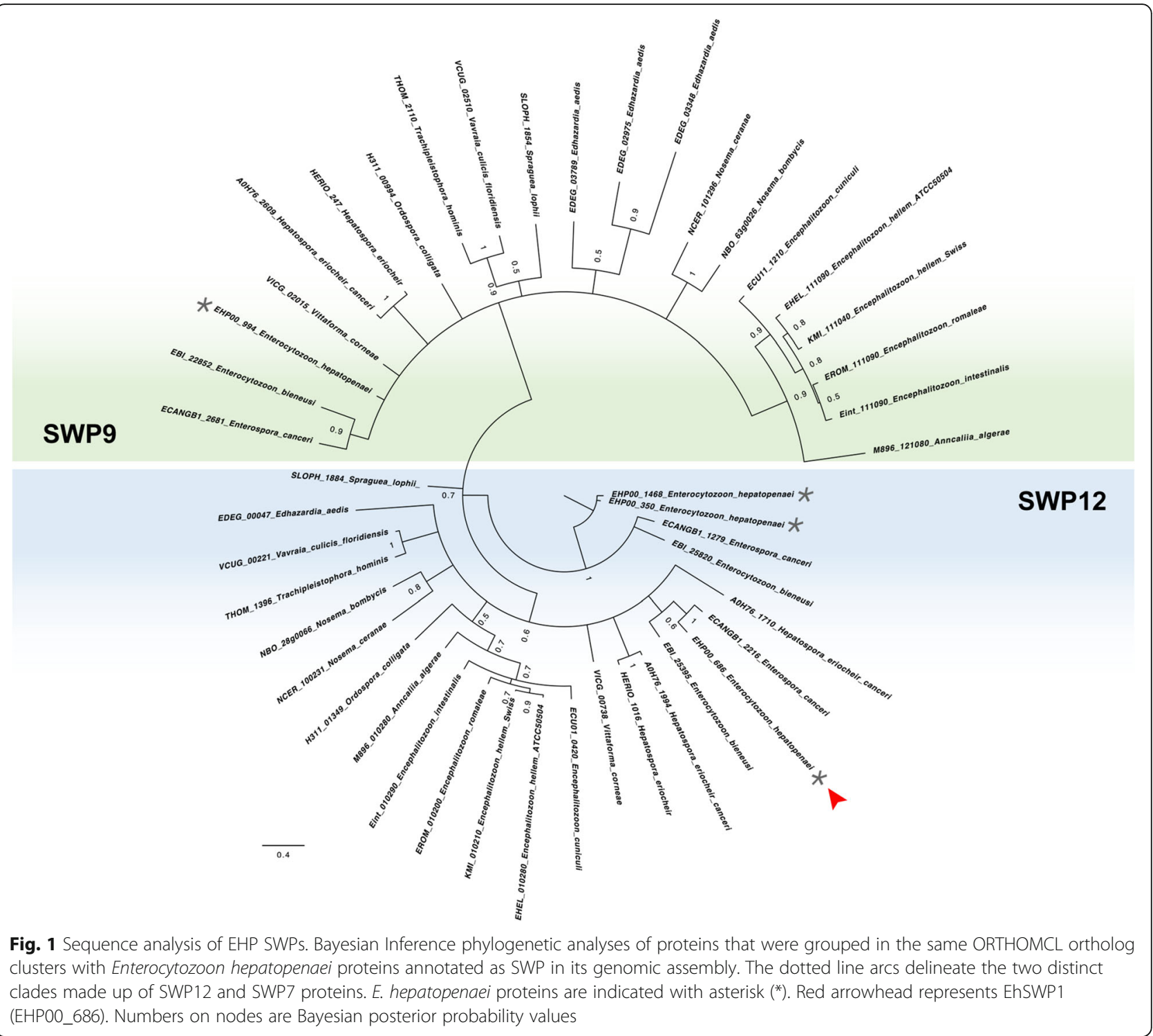

EhSWP1 was among the few proteins that were not predicted to possess any $\mathrm{O}$-glycosylation sites (see yellow stars in Fig. 2). While all proteins in the SWP12 clade were predicted to contain phosphorylation sites, none of them were positive for signal peptide sequences, GPI anchoring and transmembrane domains.

\section{Gene expression pattern of EhSWP1 during an infection}

To investigate the expression pattern of the EhSWP1 gene, single step RT-PCR analysis was performed using cDNA generated from hepatopancreatic tissue of naïve shrimp collected on days $0,5,7,9,11$ and 20 after cohabitation with EHP-infected shrimp. Positive RT-PCR amplicons for the EhSWP1 gene were detected in the naive shrimp at 20 days after the start of cohabitation (Fig. 3). However, subsequent testing using a more sensitive nested RT-PCR method revealed a low level of EhSWP1 at 11 days after cohabitation (Additional file 1: Figure S1). This indicated that a measurable level of infection was evident much earlier than 20 days and that progression of the infection was not very rapid.

\section{Immunolocalization of EhSWP1}

Purified EhSWP1-His 6 was expressed in E. coli. After induction with IPTG, a $27 \mathrm{kDa}$ overexpressed band of recombinant EhSWP1 was observed (Fig. 4a). Purification with $\mathrm{Ni}^{2+}$-NTA affinity chromatography showed that purified protein was found in fractions 2 to 5 (Fig. 4a: lanes E2-E5) after elution with $300 \mathrm{mM}$ imidazole (Fig. 4b). Later, purified protein was pooled prior to immunization of rabbits to generate polyclonal antibody against EhSWP1. Specificity of the antibody was tested 


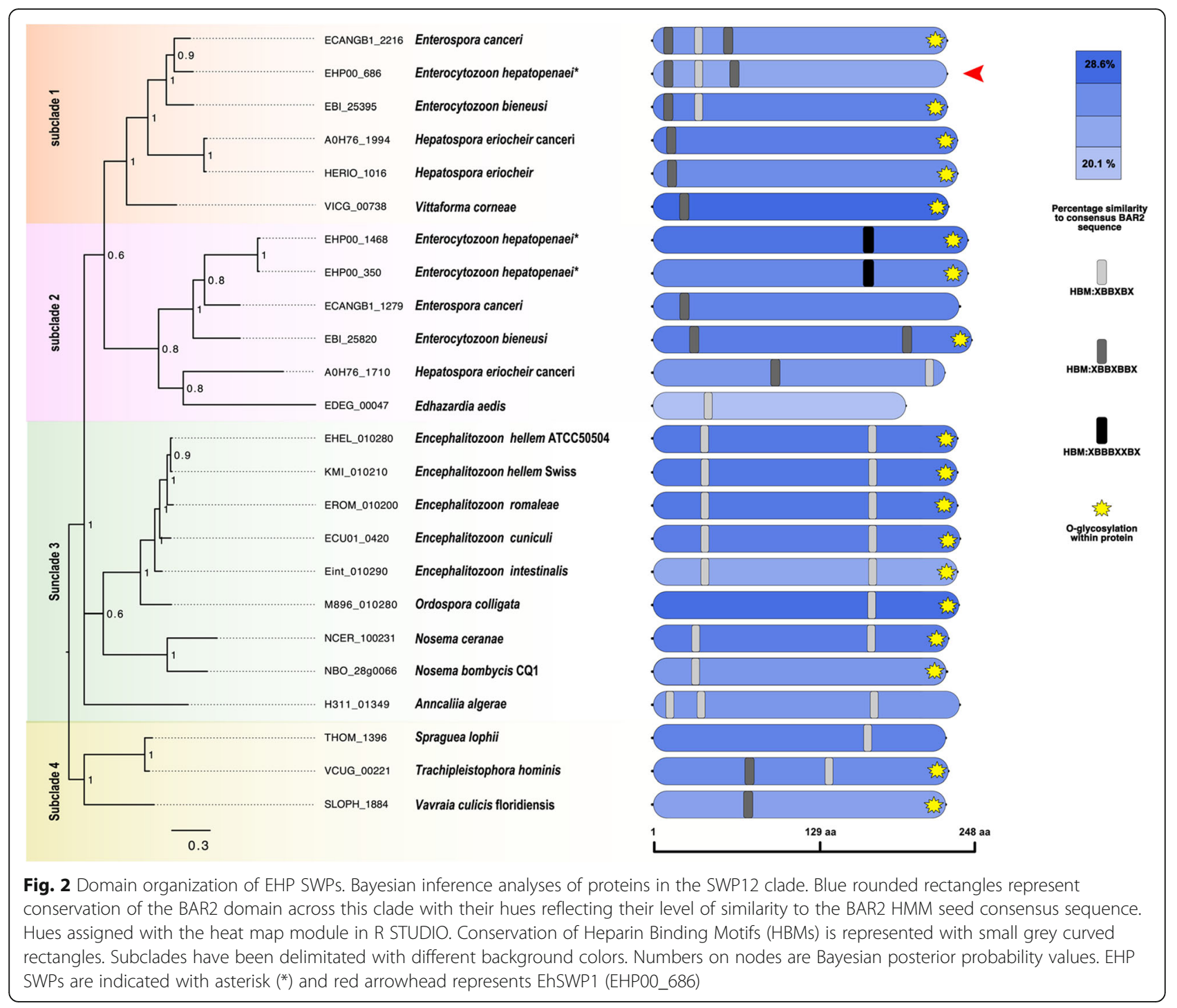

by western blot analysis (Fig. 4c). The result revealed a strong positive band at $27 \mathrm{kDa}$ that was consistent with the size of recombinant EhSWP1 (Fig. 4c). Thus, antiEhSWP1 antibody specifically bound to recombinant EhSWP1 and was suitable for localization studies.
When rabbit anti-EhSWP1 was used to perform immunofluorescence analysis (IFA) with purified spores of EHP, green fluorescence from Alexa-488 dye revealed that EhSWP1 was localized on their periphery (Fig. 5a). TO-PRO-3 dye (blue fluorescence) revealed the nucleus

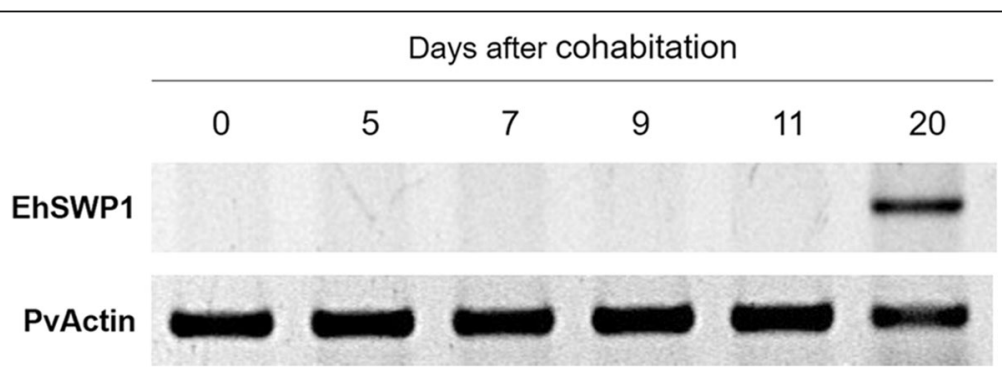

Fig. 3 EhSWP1 transcripts can be detected 20 days after cohabitation. The mRNA expression of EhSWP1 was analyzed by RT-PCR using RNA template extracted from hepatopancreatic tissue of naive shrimp cohabitated with EHP-infected shrimp. Shrimp samples were collected at 0, 5, 7, 9, 11 and 20 days after the start of cohabitation between naive shrimp and EHP-infected shrimp. The actin gene of $P$. vannamei (PvActin) was used as an internal control 


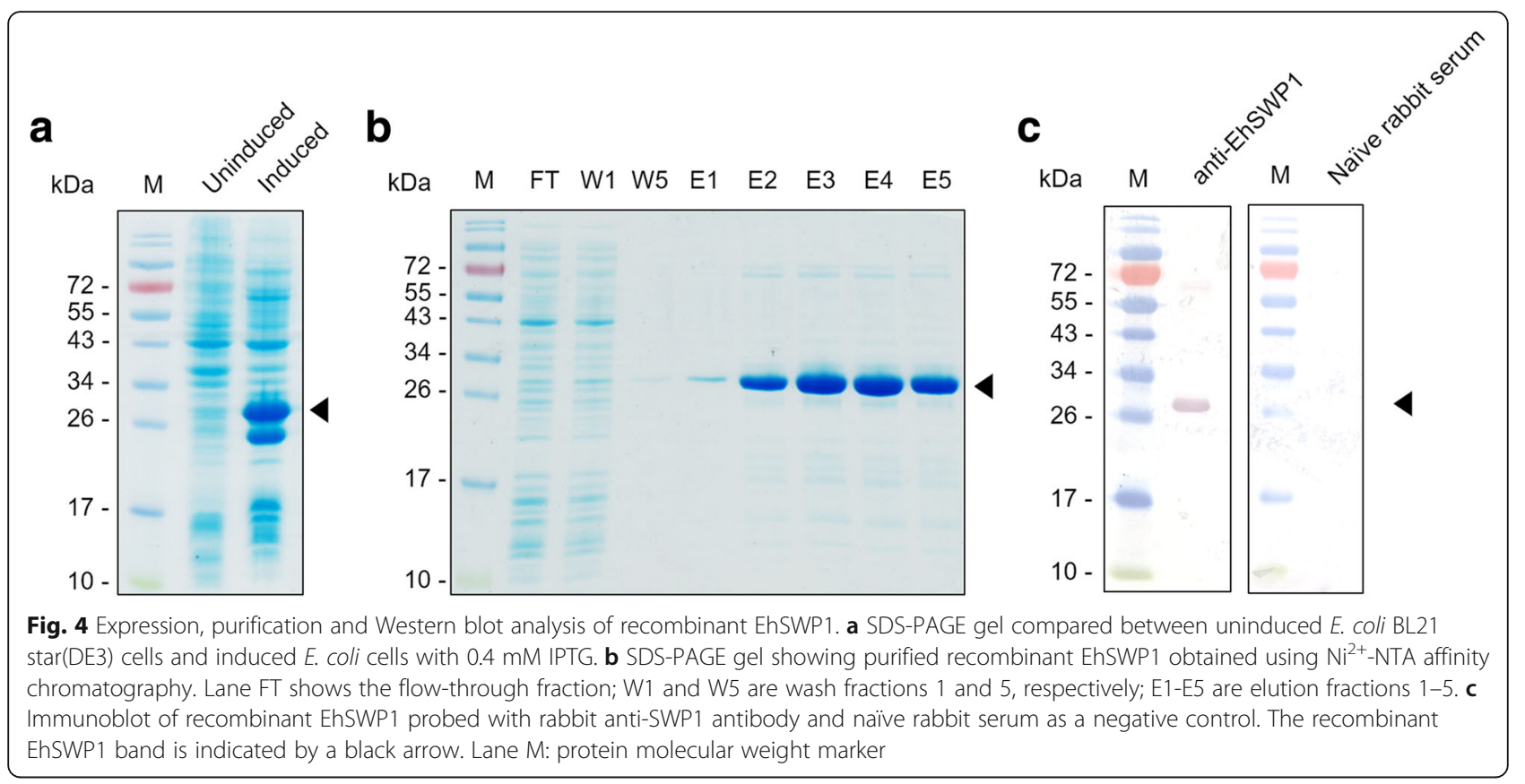

within EHP spores (Fig. 5). For the negative control group, no green fluorescence was detected (Fig. 5b). Therefore, these data confirmed that EhSWP1 was an EHP spore-wall protein.

Further immunoelectron analysis (IEM) to determine the subcellular localization of EhSWP1 revealed immunogold particles in both the exospore (Ex) and endospore layers (En), but not in the plasmalemma (Fig. 6a, b) or in the spore cytoplasm. No immunogold particles were found in the negative control group (Fig. 6c).

\section{Interaction of EhSWP1 with heparin and a competition} assay

Since sequence analysis revealed that EhSWP1 had three heparin binding motifs at its $\mathrm{N}$-terminus, preliminary assays were carried out to test its ability to bind with heparin in vitro. When recombinant EhSWP1 and BSA (Fig. 7a) were incubated with heparin beads, only recombinant EhSWP1 (but not BSA) was bound and subsequently eluted (Fig. 7b). It was possible but unlikely that the band in Fig. $7 \mathrm{~b}$ arose from a contaminant E. coli

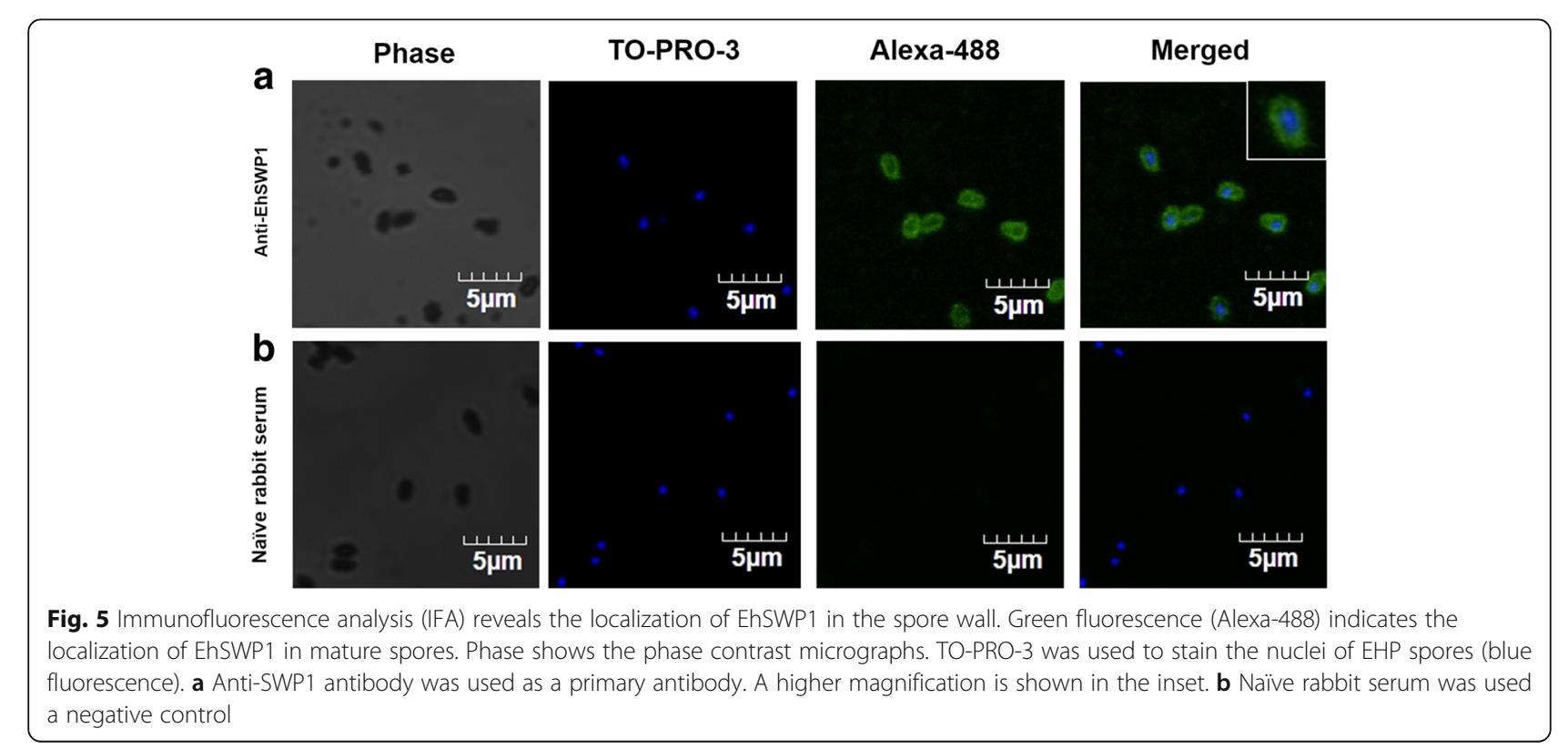



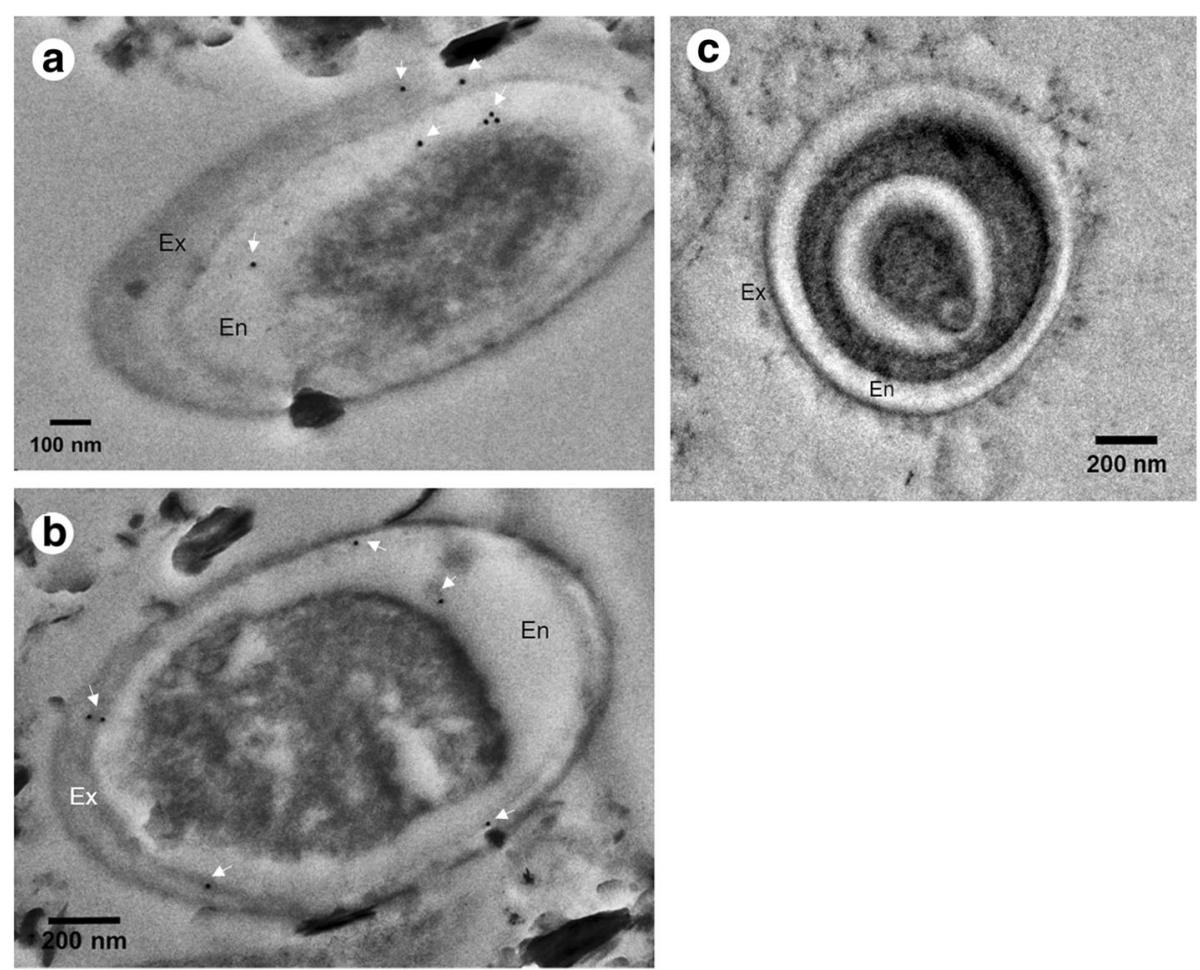

Fig. 6 Subcellular localization of EhSWP1 using Immunoelectron analysis (IEM). a, b Electron micrographs reveal the localization of EhSWP1. Exposure to anti-SWP1 antibody followed by GAR-lgG conjugated with $10 \mathrm{~nm}$ gold particles revealed immunogold particles (indicated with white arrows) indicating the presence of EhSWP1 in the exospore and endospore of EHP. c Negative control probed with naive rabbit serum shows no immunogold signals. Abbreviations: Ex, exospore layer; En, endospore layer
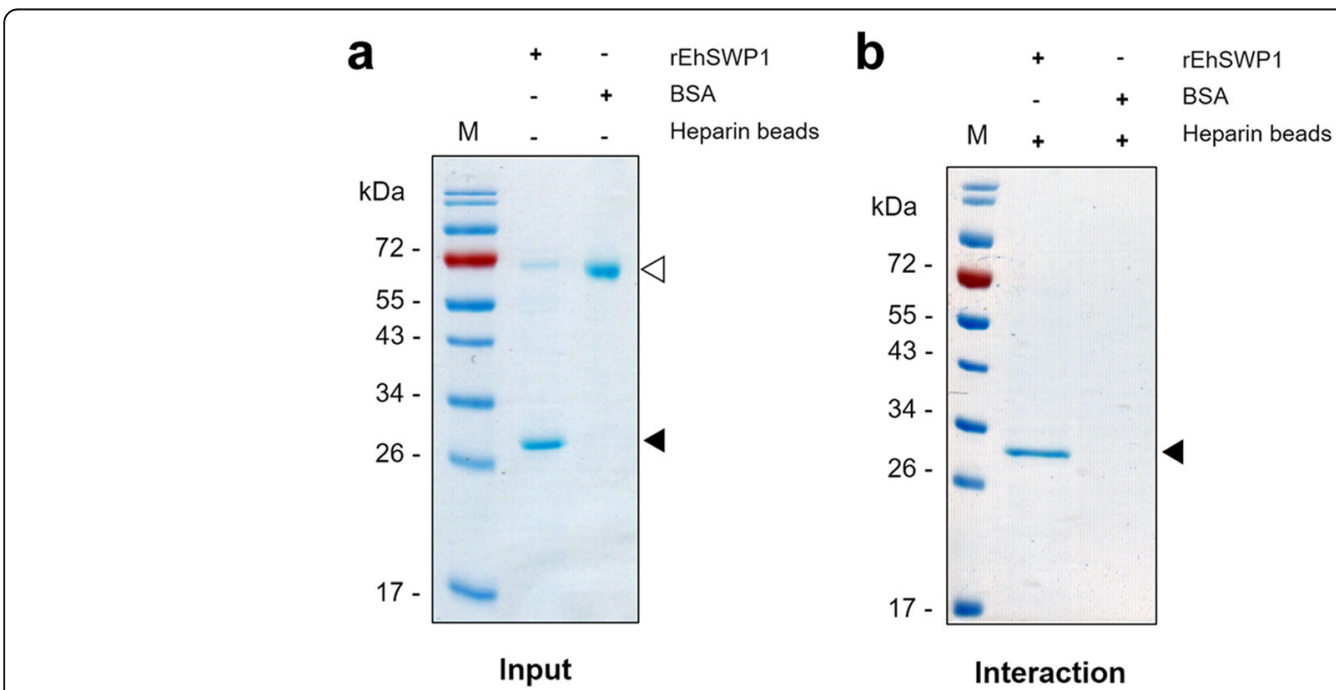

Fig. 7 Recombinant EhSWP1 binds to heparin in vitro. a SDS-PAGE gel showing input recombinant EhSWP1 (black arrow) and bovine serum albumin (BSA, white arrow) prior to mixing with heparin sepharose beads. b SDS-PAGE gel showing fractions eluted with $2 \mathrm{M} \mathrm{NaCl}$ and indicating that only EhSWP1 (black arrow) was captured and eluted from the heparin beads 
protein of the same electrophoretic mobility as recombinant EhSWP1, but this possibility was eliminated in the following experiment below.

In addition, since previous studies $[47,48]$ showed that basic residues in HBM are important for its binding activity to negatively-charged heparin, we used in vitro mutation to determine whether the function of HBM in EhSWP1 was related to heparin binding. Positively charged amino acids arginine, lysine and histidine in the three HBMs were mutated to uncharged glycine [EhSWP1 $(B \rightarrow G)]$, or to partially negative serine $[\operatorname{EhSWP1}(\mathrm{B} \rightarrow \mathrm{S})]$. Due to the substitution of larger side chains with smaller side chains, $\operatorname{EhSWP} 1(B \rightarrow G)$ and EhSWP1 $(\mathrm{B} \rightarrow \mathrm{S})$ were1-kDa lower in molecular weight than EhSWP1 wild type (EhSWP1 WT). Mutation to alanine was also carried out. However, almost all of the overexpressed alanine mutant proteins were insoluble (data not shown). Input proteins for the binding experiment are shown in Fig. 8a. After incubation of EhSWP1 WT, EhSWP1 $(B \rightarrow G)$ and EhSWP1 $(B \rightarrow S)$ with heparin beads followed by elution with $2 \mathrm{M} \mathrm{NaCl}$, only EhSWP1 WT was found in the elution fraction, not EhSWP1 $(\mathrm{B} \rightarrow \mathrm{G})$ or EhSWP1(B $\rightarrow$ S) (Fig. 8b). Western blot results using the anti-EhSWP1 antibody confirmed that only EhSWP1 WT did bind to heparin, while EhSWP1 $(\mathrm{B} \rightarrow \mathrm{G})$ and EhSWP1 $(\mathrm{B} \rightarrow \mathrm{S})$ did not (Fig. 8). This result confirmed that EhSWP1-HBMs are important for heparin binding. Since all three recombinant proteins were produced using the same E. coli expression system, the negative western blot results for EhSWP1 $(B \rightarrow G)$ or EhSWP1(B $\rightarrow$ S) (Fig. 8b) also eliminated the unlikely possibility that the band in Fig. $7 \mathrm{~b}$ and the immunopositive band in Fig. 8b arose from a contaminant $E$. coli protein of the same electrophoretic mobility as recombinant EhSWP1.

To confirm specificity of the binding, competition assays using soluble heparin were carried out. By preincubating four different concentrations of soluble heparin with recombinant EhSWP1 prior to mixing with heparin-sepharose beads, it was shown that $10 \mathrm{mg} / \mathrm{ml}$ of soluble heparin could reduce the binding by more than 40\% (Fig. 9, Additional file 2: Figure S2). Increasing the soluble heparin to $100 \mathrm{mg} / \mathrm{ml}$ reduced the binding by $84 \%$ (Fig. 9c). However, there was no reduction in binding when there was no exogenous heparin or heparin at $0.1 \mathrm{mg} / \mathrm{ml}$ (Fig. 9c). This result suggests that exogenous heparin can inhibit the interaction of EhSWP1 with heparin in a dose dependent manner and that heparin is indeed an EhSWP1 binding partner.

\section{Discussion}

Diversity and phylogeny of spore wall proteins

The microsporidian infection process is unique compared to that of other intracellular parasites $[49,50]$. Their spores possess a special organelle called a polar tube that is extruded to pierce host cell membranes and
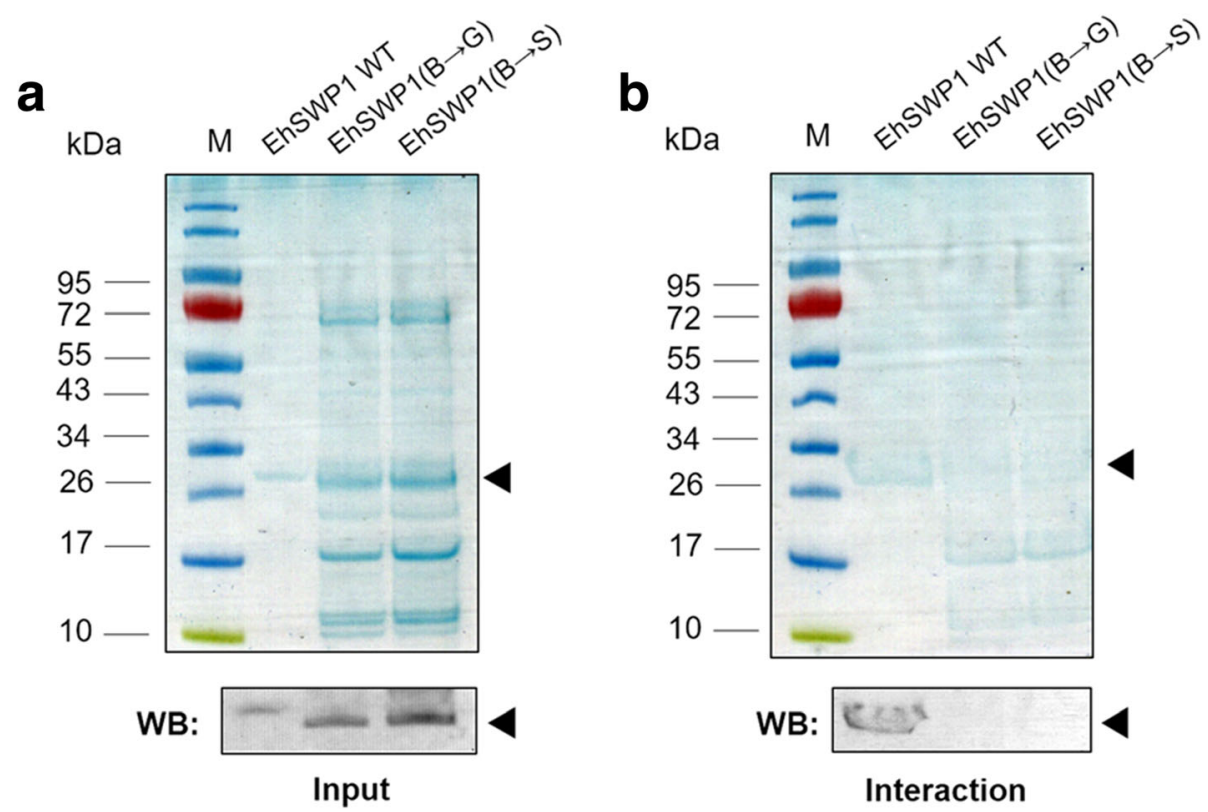

Fig. 8 EhSWP1-HBM mutants fail to bind to heparin beads. a SDS-PAGE gel showing input proteins EhSWP1 WT, EhSWP1(B $\rightarrow G)$ and EhSWP1(B $\rightarrow$ S) (black arrow) with molecular weights of $27 \mathrm{kDa}, 26 \mathrm{kDa}$ and $26 \mathrm{kDa}$, respectively. b SDS-PAGE gel showing elution fractions after incubation with heparin sepharose beads and revealing that only EhSWP1 WT (black arrow) was captured and eluted from the beads. Lower panels (indicated as WB) are western blots probed with anti-EhSWP1 antibody to confirm protein identity as EhSWP1 (black arrows) 

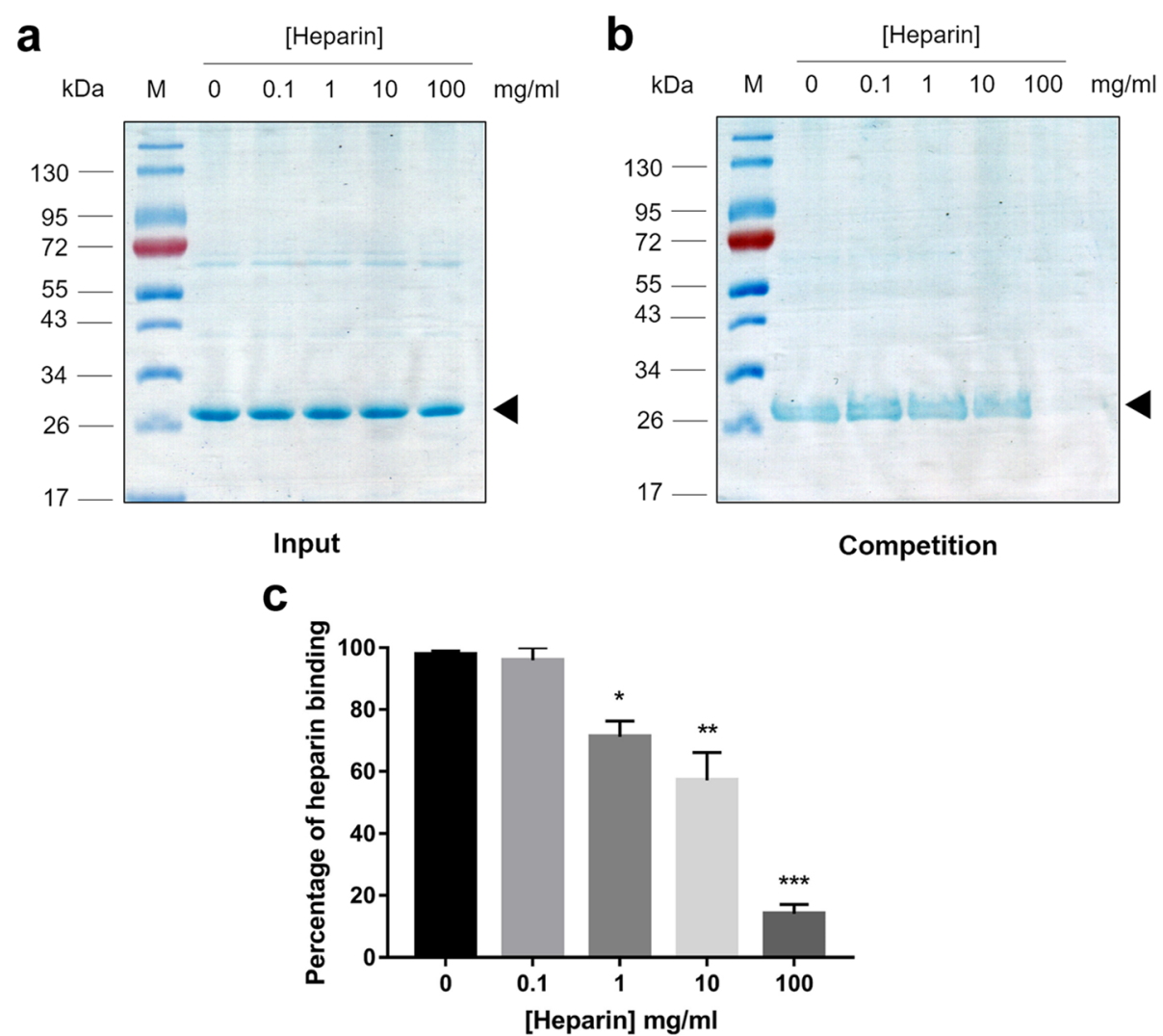

Fig. 9 Heparin competition assay. a SDS-PAGE gel showing input recombinant EhSWP1 (black arrow) with different concentrations of soluble porcine heparin. $\mathbf{b}$ SDS-PAGE gel shows elution fractions after $1 \mathrm{~h}$ competition and revealing that binding of EhSWP1 (black arrow) to heparin beads was blocked at $100 \mathrm{mg} / \mathrm{ml}$. c Bar graph showing percentage of heparin binding quantified from the protein bands from 3 with replicates gels (Additional file 2: Figure S2). Error bars indicate the mean \pm SEM. Level of heparin binding at $0 \mathrm{mg} / \mathrm{ml}$ was used for normalization. ${ }^{*} P \leq 0.01$; ${ }^{* *} P \leq 0.001 ; * * P \leq 0.0001$

serve as a conduit to transfer the infectious spore contents (sporoplasm) into the host cell cytoplasm [16]. However, microsporidia require relatively close proximity to host cells for the first step of infection [17, 45]. Previous studies have revealed that SWPs are important in the attachment of microsporidian spores to their hosts $[17,18]$.

Orthology clustering and phylogenetic analyses performed in this study identified the four proteins annotated as SWPs in EHPs genomic assembly [40] to fall under two distinct clades of microsporidian SWPs, SWP12 and SWP7. Signature sequences of HBMs are well characterized, namely XBBXBX, XBBXBBX, XBBBXXBX and XBBB XXBBBXXBBX, where $\mathrm{X}$ represents a hydrophobic amino acid and $B$ represents a positively charged amino acid $[48,51,52]$. Although XBBXBX and XBBXBBX HBMs appeared to be highly conserved across the SWP12 clade in our analysis, their exact positioning and enrichment was specific to microsporidian families and sometimes, species (Fig. 2). In light of the importance of SWP HBMs in parasite-host tethering [18, 24], this family/species-specific HBM positioning and enrichment perhaps reflect the different host environs and cell types with which these proteins have evolved to interact. Our phylogenetic analysis suggests there was a duplication of the SWP12 gene in the common ancestor of species belonging to the family Enterocytozoonidae, with positional conservation of HBMs only being maintained in subclade 1 (Fig. 2). This duplication event, unique to the Enterocytozoonidae, hints at the importance of this particular protein in the life-cycle of species within this family. Gene duplication is known to facilitate innovation in genomes by allowing the duplicate gene to develop new functional properties via the accruement of non-deleterious mutations, a process referred to as neofunctionalization. Finally, our analyses corroborated previous research that predicted NbSWP12 (NBO_28g0066) and E. intestinalis EnP1 to contain 1 and 2 HBMs, respectively $[18,24]$.

Our alignment results suggest that the BAR2 domain is conserved across all proteins that clustered within the SWP12 clade. Known functions of this domain include 
membrane shaping and signalling control processes, but its role in microsporidian proteins is yet to be elucidated [53]. The conservation of this domain in the SWP12 clade, however, alludes to its importance in the function of SWP12 proteins [24].

\section{Expression profiles of spore wall proteins}

Expression profiles of SWPs vary in different microsporidian species. Feeding of fourth instar silkworm larvae with mulberry leaves contaminated with $N$. bombycis spores showed that NbSWP5, NbSWP12 and NbSWP15 were expressed on day 3 post-infection [22, 24, 25]. In contrast, transcripts of NbSWP11 appeared at a low level on day 1 post-infection and gradually rose until day 7 [23]. Moreover, starvation treatment of third instar nymph locusts followed by feeding with $A$. locustae spores revealed that AlocSWP2 expression was detected on day 9 after spore inoculation [30]. Our cohabitation study between naïve shrimp and EHP-infected shrimp showed that EhSWP1 transcripts were observed only at 20 days after the start of cohabitation. However, by using RT-PCR followed by nested-PCR analysis specific to the EhSWP1 gene, a low level of expression was found at 11 days after cohabitation. The result may suggest that EHP requires at least 11 days to develop into mature spores. However, this needs to be confirmed by other analyses.

\section{EhSWP1: its role in host-cell tethering}

Heparin is a member of the GAG family and has been extensively studied in vertebrate species. A major function of heparin is to serve as a blood anticoagulant [54]. It is also used as an antithrombotic agent against heart and vascular thrombosis [55]. In mammals, heparin is mainly distributed in the lungs, intestine and liver [56]. Heparin is not only found in vertebrates, but also in invertebrates including crustaceans, molluscs, annelids, echinoderms and cnidarians [57]. However, there are very few studies on localization of heparin in organs and cell types. In the northern quahog clam, heparin was found at the proximal to epithelial surfaces of cells in the intestine, palp and siphon [58]. For shrimp, there has been no study on heparin distribution. However, heparin has been successfully extracted from the cephalothorax (where the gills, heart, intestine and hepatopancreas are located [59]) in the red-spotted shrimp P. brasilliensis and the Pacific white shrimp P. vannamei $[60,61]$. Transcriptomic analysis of the hepatopancreas of $P$. vannamei showed that genes involved in the GAG biosynthesis pathway were active [62] and suggested that heparin might be present in the hepatopancreas. In this study, we showed that EhSWP1 could bind to heparin using the in vitro heparin binding assay. From immunofluorescence and immunoelectron analyses of EHP spores, we also showed that EhSWP1 is localized in both the exospore and endospore layers, similar to what has been previously described for SWPs from other microsporidians including EiEnP1, NbSWP9 and NbSWP26 $[18,27,63]$. The results support our hypothesis that EHP uses EhSWP1 to bind to heparin of target cells in shrimp hepatopancreatic tissue (Fig. 10) [33, 34].

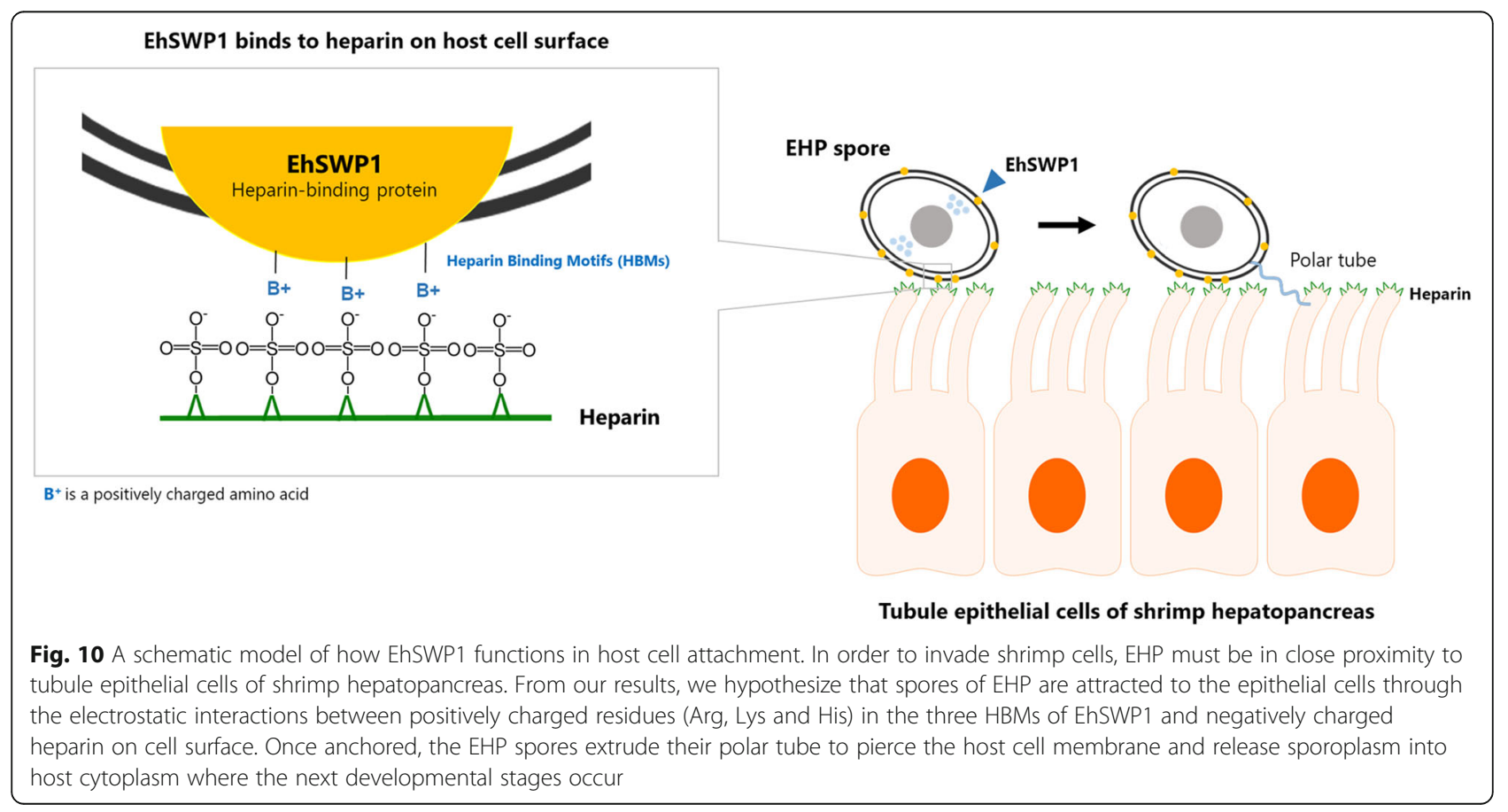


It is not only EHP that utilizes heparin for attachment to host cells. Other intracellular parasites such as Trypanosoma cruzi also use heparin-binding proteins for host cell recognition. Incubation of its epimastigote stage with heparin and heparin sulfate can inhibit parasite binding to immobilized heparin and also inhibit parasite binding to midgut epithelial cells of their insect vectors [64]. In the malarial parasite Plasmodium falciparum, BAEBL protein binding to erythrocytes was disrupted by addition of soluble heparin [65]. The competition assay presented here showed that soluble heparin inhibited interaction between EhSWP1 and immobilized heparin beads in a dose dependent manner and suggests that heparin would inhibit EhSWP1 binding to shrimp host cells via their surface heparin.

Since there is no EHP infection model in hepatopancreatic cell cultures or any immortal shrimp cell line, in vivo tests of spore adherence could not be carried out but should constitute a future goal to confirm whether exogenous soluble heparin could reduce or inhibit EHP spore attachment to host cells. Similar tests would also show whether or not the antibody against EhSWP1 could reduce spore adherence. From previous studies, anti-EcEnP1 antibody inhibited spore adherence by $56 \%$ [18], while anti-NbSWP16 antibody reduced adherence by $20 \%$ [25]. Such in vivo assays with host cells are required to fully understand the function of EhSWP1.

\section{Conclusions}

In summary, this is the first report on characterization of a spore wall protein from the microsporidian $E$. hepatopenaei (EhSWP1). It is present in both the exospore and endospore layers of mature spore walls and it has been shown to bind with heparin, indicating a possible role in attachment to host cells via surface heparin as an early step in the host cell infection process and constituting an important role in virulence (Fig. 10). This knowledge may lead to the development of novel therapeutics to combat to EHP infection.

\section{Additional files}

Additional file 1: Figure S1. Transcriptional pattern of EhSWP1 using one-step RT-PCR and nested RT-PCR analysis of RNA template from naïve shrimp cohabitated with EHP-infected shrimp. (TIFF $497 \mathrm{~kb}$ )

Additional file 2: Figure S2. Experimental replicates of the heparin competition assay. (a) replicate II and (b) replicate III. (TIFF $984 \mathrm{~kb}$ )

\section{Abbreviations}

EHP: Enterocytozoon hepatopenaei; EhSWP1: Spore wall protein 1 of EHP; HBM: Heparin binding motif; ORF: Open reading frame; ppt: parts per trillion

\section{Acknowledgements}

The authors would like to thank Professor T. W. Flegel for assistance in designing the study, supervising the project and editing the manuscript, and Asst. Professor Danaya Pakotiprapha for providing heparin sepharose beads and giving very useful comments.

\section{Funding}

This project was supported by the Agricultural Research Development Agency (ARDA) of Thailand under project CRP5905020530, by the Thailand Research Fund (TRF) under project IRG5980008 and TRG5780032, by the Newton Institutional Links (IL) program to BIOTEC, Thailand and Cefas, UK, and by Mahidol University. PJ would like to thank the Science Achievement Scholarship of Thailand (SAST) for a PhD scholarship.

\section{Availability of data and materials}

All data generated or analysed during this study are included in this published article and its additional files.

\section{Authors' contributions}

PJ, Ol and KS designed the study. OI and KS obtained the funding. PJ and RV performed experiments. DWB and BAPW performed bioinformatics analyses. $\mathrm{OI}, \mathrm{KS}$ and RV supervised the project. PJ, DWB and OI wrote and edited the manuscript. All authors read and approved the final manuscript.

\section{Ethics approval and consent to participate}

At the time this work was carried out, there was no official standard of the Ethical Principles and Guidelines for the Use of Animals of the National Research Council of Thailand (1999) for invertebrates. However, its principles for vertebrates were adapted for shrimp. The guidelines of the New South Wales State Government (Australia) for the humane harvesting of fish and crustaceans were also followed (https:/www.dpi.nsw.gov.au/animals-and-livestock/animalwelfare/general/welfare-of-fish/shellfish) with respect to details regarding the transport of the shrimp and their laboratory maintenance. With respect to processing of the shrimp for histological analysis or for killing at the end of an experiment, the saltwater/ice slurry method was used as recommended in the Australian guidelines.

\section{Consent for publication}

Not applicable.

\section{Competing interests}

The authors declare that they have no competing interests.

\section{Publisher's Note}

Springer Nature remains neutral with regard to jurisdictional claims in published maps and institutional affiliations.

\section{Author details}

${ }^{1}$ Department of Biochemistry, Faculty of Science, Mahidol University, Bangkok, Thailand. ${ }^{2}$ Center of Excellence for Shrimp Molecular Biology and Biotechnology (Centex Shrimp), Faculty of Science, Mahidol University, Bangkok, Thailand. ${ }^{3}$ Biosciences, College of Life and Environmental Sciences, University of Exeter, Devon, UK. ${ }^{4}$ Department of Anatomy, Faculty of Science, Mahidol University, Bangkok, Thailand. ${ }^{5}$ National Center for Genetic Engineering and Biotechnology (BIOTEC), National Science and Technology Development Agency (NSTDA), Pathumthani, Thailand. ${ }^{6}$ Shrimp Pathogen Interaction Laboratory (SPI), National Center for Genetic Engineering and Biotechnology (BIOTEC), Bangkok, Thailand.

Received: 6 December 2017 Accepted: 28 February 2018 Published online: 12 March 2018

\section{References}

1. Choi J, Kim SH. A genome tree of life for the Fungi kingdom. Proc Natl Acad Sci USA. 2017;114:9391-6.

2. Keeling PJ, Fast NM. Microsporidia: biology and evolution of highly reduced intracellular parasites. Annu Rev Microbiol. 2002;56:93-116.

3. Stentiford GD, Feist SW, Stone DM, Bateman KS, Dunn AM. Microsporidia: diverse, dynamic, and emergent pathogens in aquatic systems. Trends Parasitol;: 2013;29:567-78.

4. Nägeli KW. Über die neue krankheit der seidenraupe und verwandte organismen. Bot Z. 1857;15:760-1.

5. Bhat SA, Bashir I, Kamili AS. Microsporidiosis of silkworm, Bombyx mori L. (Lepidoptera: Bombycidae): a review. African J Agric Res. 2009;4:1519-23.

6. Becnel JJ, Andreadis TG. Microsporidia in insects. In: Weiss LM, Becnel JL, editors. Microsporidia: Pathogens of Opportunity. First Ed. West Sussex: Wiley Blackwell; 2014. p. 521-570. 
7. Kotler DP, Orenstein JM. Clinical syndromes associated with microsporidiosis. Adv Parasitol. 1998;40:321-49.

8. Rodriguez-Tovar LE, Speare DJ, Markham RJF. Fish microsporidia: immune response, immunomodulation and vaccination. Fish Shellfish Immunol. 2011;30:999-1006.

9. Thitamadee S, Prachumwat A, Srisala J, Jaroenlak P, Salachan PV, Sritunyalucksana $\mathrm{K}$, et al. Review of current disease threats for cultivated penaeid shrimp in Asia. Aquaculture. 2016;452:69-87.

10. Takvorian PM, Cali A. Enzyme histochemical identification of the Golgi apparatus in the microsporidian, Glugea stephani. J Eukaryot Microbiol. 1993:41:63-4.

11. Franzen C. Microsporidia: how can they invade other cells? Trends Parasitol. 2004:20:275-9.

12. Williams BAP, Hirt RP, Lucocq JM, Embley TM. A mitochondrial remnant in the microsporidian Trachipleistophora hominis. Nature. 2002;418:865-9.

13. Burri L, Williams BAP, Bursac D, Lithgow T, Keeling PJ. Microsporidian mitosomes retain elements of the general mitochondrial targeting system Proc Natl Acad Sci USA. 2006;103:15916-20.

14. Peyretaillade E, El Alaoui H, Diogon M, Polonais V, Parisot N, Biron DG, et al. Extreme reduction and compaction of microsporidian genomes. Res Microbiol. 2011;162:598-606.

15. Corradi N, Pombert J-F, Farinelli L, Didier ES, Keeling PJ. The complete sequence of the smallest known nuclear genome from the microsporidian Encephalitozoon intestinalis. Nat Commun. 2010;1:77-84.

16. Weidner E. The microsporidian spore invasion tube. the ultrastructure, isolation, and characterization of the protein comprising the tube. J Cell Biol. 1976;71:23-34

17. Hayman JR, Southern TR, Nash TE. Role of sulfated glycans in adherence of the microsporidian Encephalitozoon intestinalis to host cells in vitro. Infect Immun. 2005;73:841-8.

18. Southern TR, Jolly CE, Lester ME, Hayman JR. EnP1, a microsporidian spore wall protein that enables spores to adhere to and infect host cells in vitro. Eukaryot Cell. 2007;6:1354-62.

19. Frixione E, Ruiz L, Cerbón J, Undeen AH. Germination of Nosema algerae (Microspora) spores: conditional inhibition by D2O, ethanol and $\mathrm{Hg} 2+$ suggests dependence of water influxupon membrane hydration and specific transmembrane pathways. J Eukaryot Microbiol. 1997;44:109-16.

20. Vávra J, Lukeš J. Microsporidia and "the art of living together". Adv Parasitol. 2013;82:253-319.

21. Weiss LM, Delbac F, Hayman JR, Pan G, Dang X, Zhou Z. The microsporidian polar tube and spore wall. In: Weiss LM, Becnel JL, editors. Microsporidia: Pathogens of Opportunity. First Ed. West Sussex, UK: Wiley Blackwell; 2014. p. 261-306

22. Li Z, Pan G, Li T, Huang W, Chen J, Geng L, et al. SWP5, a spore wall protein, interacts with polar tube proteins in the parasitic microsporidian Nosema bombycis. Eukaryot Cell. 2012;11:229-37.

23. Yang $\mathrm{D}$, Dang $\mathrm{X}$, Peng $\mathrm{P}$, Long $\mathrm{M}, \mathrm{Ma} \mathrm{C}$, Qin JJG, et al. NbHSWP11, a microsporidia Nosema bombycis protein, localizing in the spore wall and membranes, reduces spore adherence to host cell BME. J Parasitol. 2014; 100:623-32.

24. Chen J, Geng L, Long M, Li T, Li Z, Yang D, et al. Identification of a novel chitin-binding spore wall protein (NbSWP12) with a BAR-2 domain from Nosema bombycis (microsporidia). Parasitology. 2013;140:1394-402.

25. Wang Y, Dang X, Ma Q, Liu F, Pan G, Li T, et al. Characterization of a novel spore wall protein NbSWP16 with proline-rich tandem repeats from Nosema bombycis (microsporidia). Parasitology. 2014;142:534-42.

26. Wu Z, Li Y, Pan G, Zhou Z, Xiang Z. SWP25, a novel protein associated with the Nosema bombycis endospore. J Eukaryot Microbiol. 2009;56:113-8.

27. Li Y, Wu Z, Pan G, He W, Zhang R, Hu J, et al. Identification of a novel spore wall protein (SWP26) from microsporidia Nosema bombycis. Int J Parasitol. 2009:39:391-8.

28. Peuvel-Fanget I, Polonais V, Brosson D, Texier C, Kuhn L, Peyret $P$, et al. EnP1 and EnP2, two proteins associated with the Encephalitozoon cuniculi endospore, the chitin-rich inner layer of the microsporidian spore wall. Int J Parasitol. 2006;36:309-18.

29. Brosson $D$, Kuhn $L$, Prensier $G$, Vivarès $C P$, Texier $C$. The putative chitin deacetylase of Encephalitozoon cuniculi: a surface protein implicated in microsporidian spore-wall formation. FEMS Microbiol Lett. 2005;247:81-90.

30. Chen L, Li R, You Y, Zhang K, Zhang L. A novel spore wall protein from Antonospora locustae (Microsporidia: Nosematidae) contributes to sporulation. J Eukaryot Microbiol. 2017;64:779-91.
31. Jaroenlak P, Sanguanrut P, Williams BAP, Stentiford GD, Flegel TW, Sritunyalucksana $\mathrm{K}$, et al. A nested PCR assay to avoid false positive detection of the microsporidian Enterocytozoon hepatopenaei (EHP) in environmental samples in shrimp farms. PLoS One. 2016;11:e0166320.

32. Sritunyalucksana K, Sanguanrut P, Salachan PV, Thitamadee S, Flegel TW. Urgent appeal to control spread of the shrimp microsporidian parasite Enterocytozoon hepatopenaei (EHP). Network of Aquaculture Centres in Asia-Pacific (NACA). 13 Dec 2014;4-6. enaca.org/publications/health/diseasecards/urgent-appeal-to-control-ehp-3.pdf

33. Chayaburakul K, Nash G, Pratanpipat P, Sriurairatana S, Withyachumnarnkul B. Multiple pathogens found in growth-retarded black tiger shrimp Penaeus monodon cultivated in Thailand. Dis Aquat Org. 2004;60:89-96.

34. Tourtip S, Wongtripop S, Stentiford GD, Bateman KS, Sriurairatana S, Chavadej J, et al. Enterocytozoon hepatopenaei sp. nov. (Microsporida: Enterocytozoonidae), a parasite of the black tiger shrimp Penaeus monodon (Decapoda: Penaeidae): fine structure and phylogenetic relationships. I Invertebr Pathol. 2009;102:21-9.

35. Tangprasittipap A, Srisala J, Chouwdee S, Somboon M, Chuchird N, Limsuwan C, et al. The microsporidian Enterocytozoon hepatopenaei is not the cause of white feces syndrome in whiteleg shrimp Penaeus (Litopenaeus) vannamei. BMC Vet Res. 2013;9:139.

36. Tang KFJ, Pantoja CR, Redman RM, Han JE, Tran LH, Lightner DV. Development of in situ hybridization and PCR assays for the detection of Enterocytozoon hepatopenaei (EHP), a microsporidian parasite infecting penaeid shrimp. J Invertebr Pathol. 2015;130:37-41.

37. Rajendran KV, Shivam S, Ezhil PP, Joseph SRJ, Sathish KT, Avunje S, et al. Emergence of Enterocytozoon hepatopenaei (EHP) in farmed Penaeus (Litopenaeus) vannamei in India. Aquaculture. 2016:454:272-80.

38. Tang KFJ, Aranguren LF, Piamsomboon P, Han JE, Maskaykina IY, Schmidt MM. Detection of the microsporidian Enterocytozoon hepatopenaei (EHP) and Taura syndrome virus in Penaeus vannamei cultured in Venezuela. Aquaculture. 2017:480:17-21.

39. Salachan PV, Jaroenlak P, Thitamadee S, Itsathitphaisarn O, Sritunyalucksana K. Laboratory cohabitation challenge model for shrimp hepatopancreatic microsporidiosis (HPM) caused by Enterocytozoon hepatopenaei (EHP). BMC Vet Res. 2016;13:9.

40. Wiredu Boakye D, Jaroenlak P, Prachumwat A, Williams TA, Bateman KS, Itsathitphaisarn O, et al. Decay of the glycolytic pathway and adaptation to intranuclear parasitism within Enterocytozoonidae microsporidia. Environ Microbiol. 2017;19:2077-89.

41. Camacho C, Coulouris G, Avagyan V, Ma N, Papadopoulos J, Bealer K, et al. BLAST+: architecture and applications. BMC Bioinformatics. 2009;10:421.

42. Li L, Stoeckert CJJ, Roos DS. OrthoMCL: Identification of ortholog groups for eukaryotic genomes. Genome Res. 2003;13:2178-89.

43. Ronquist F, Teslenko M, Van Der Mark P, Ayres DL, Darling A, Höhna S, et al. MrBayes 3.2: efficient Bayesian phylogenetic inference and model choice across a large model space. Syst Biol. 2012;61:539-42.

44. Somchai P, Jitrakorn S, Thitamadee S, Meetam M, Saksmerprome V. Use of microalgae Chlamydomonas reinhardtii for production of double-stranded RNA against shrimp virus. Aquac Reports. 2016;3:178-83.

45. Southern TR, Jolly CE, Hayman JR. Augmentation of microsporidia adherence and host cell infection by divalent cations. FEMS Microbiol Lett. 2006;260:143-9.

46. Yang D, Pan L, Peng P, Dang X, Li C, Li T, et al. Interaction between SWP9 and PTPs of the microsporidian Nosema bombycis and SWP9 as a scaffolding protein contributes to the polar tube tethering to spore wall. Infect Immun. 2016;85:00872-16.

47. Proudfoot AEl, Fritchley S, Borlat F, Shaw JP, Vilbois F, Zwahlen C, et al. The BBXB motif of RANTES is the principal site for heparin binding and controls receptor selectivity. J Biol Chem. 2001;276:10620-6.

48. Marinò M, Friedlander JA, McCluskey RT, Andrews D. Identification of a heparin-binding region of rat thyroglobulin involved in megalin binding. J Biol Chem. 1999;274:30377-86.

49. Bigliardi E, Sacchi L. Cell biology and invasion of the microsporidia. Microbes Infect. 2001;3:373-9.

50. Weiss LM, Becnel JJ. Pathogens of Opportunity. First Ed. West Sussex, UK: Wiley. Blackwell. 2014;

51. Cardin AD, Weintraub HJ. Molecular modeling of protein-glycosaminoglycan interactions. Arteriosclerosis. 1989;9:21-32.

52. Capila I, Linhardt RJ. Heparin-protein interactions. Angew Chemie. 2002:41: $390-412$. 
53. Kessels MM, Qualmann B. Different functional modes of BAR domain proteins in formation and plasticity of mammalian postsynapses. J Cell Sci. 2015;128:3177-85.

54. Linhardt RJ. 2003 Claude S. Hudson Award Address in Carbohydrate Chemistry. Heparin: structure and activity. J Med Chem. 2003;46:2551-64.

55. Arias E, Smith BL. Deaths: preliminary data for 2001. Natl Vital Stat Rep. 2003: 51:1-44.

56. Nader HB, Lopes CC, Rocha HAO, Santos EA, Dietrich CP. Heparins and heparinoids: occurrence, structure and mechanism of antithrombotic and hemorrhagic activities. Curr Pharm Des. 2004;10:951-66.

57. Volpi N. Occurrence and structural characterization of heparin from molluscs. ISJ. 2005;2:6-16.

58. Ulrich PN, Boon JK. The histological localization of heparin in the northern quahog clam, Mercenaria mercenaria. J Invertebr Pathol. 2001;78:155-9.

59. Bell TA, Lightner DV. A handbook of normal penaeid shrimp histology. Baton Rouge: World Aquaculture Society; 1988.

60. Brito AS, Cavalcante RS, Palhares LC, Hughes AJ, Andrade GP, Yates EA, et al. A non-hemorrhagic hybrid heparin/heparan sulfate with anticoagulant potential. Carbohydr Polym. 2014;99:372-8.

61. Dietrich CP, Paiva JF, Castro RAB, Chavante SF, Jeske W, Fareed J, et al. Structural features and anticoagulant activities of a novel natural low molecular weight heparin from the shrimp Penaeus brasiliensis. Biochim Biophys Acta-Gen Subj. 1999;1428:273-83.

62. Chen K, Li E, Li T, Xu C, Wang X, Lin H, et al. Transcriptome and molecular pathway analysis of the hepatopancreas in the Pacific white shrimp Litopenaeus vannamei under chronic low-salinity stress. PLoS One. 2015;10:1-22.

63. Yang D, Pan G, Dang X, Shi Y, Li C, Peng P, et al. Interaction and assembly of two novel proteins in the spore wall of the microsporidian species Nosema bombycis and their roles in adherence to and infection of host cells. Infect Immun. 2015;83:1715-31.

64. Oliveira FOR, Alves CR, Souza-Silva F, Calvet CM, Côrtes LMC, Gonzalez MS, et al. Trypanosoma cruzi heparin-binding proteins mediate the adherence of epimastigotes to the midgut epithelial cells of Rhodnius prolixus. Parasitology. 2012;139:735-43.

65. Kobayashi K, Kato K, Sugi T, Takemae H, Pandey K, Gong H, et al. Plasmodium falciparum BAEBL binds to heparan sulfate proteoglycans on the human erythrocyte surface. J Biol Chem. 2010;285:1716-25.

\section{Submit your next manuscript to BioMed Central and we will help you at every step:}

- We accept pre-submission inquiries

- Our selector tool helps you to find the most relevant journal

- We provide round the clock customer support

- Convenient online submission

- Thorough peer review

- Inclusion in PubMed and all major indexing services

- Maximum visibility for your research

Submit your manuscript at www.biomedcentral.com/submit

) Biomed Central 